\title{
Biomechanical mechanism for transitions in phase and frequency of arm and leg swing during walking
}

\author{
Masayoshi Kubo $^{1}$, Robert C. Wagenaar ${ }^{2}$, Elliot Saltzman ${ }^{2}$, Kenneth G. Holt ${ }^{2}$ \\ ${ }^{1}$ Division of Kinesiology, University of Michigan, 401 Washtenaw Ave., Ann Arbor, MI 48109-2214, USA \\ ${ }^{2}$ Department of Rehabilitation Sciences, Sargent College of Health and Rehabilitation Sciences, Boston University, \\ 635 Commonwealth Ave. Boston, MA 02215, USA
}

Received: 5 May 2003 / Accepted: 1 July 2004 / Published online: 24 August 2004

\begin{abstract}
As humans increase walking speed, there are concurrent transitions in the frequency ratio between arm and leg movements from 2:1 to $1: 1$ and in the phase relationship between the movements of the two arms from in-phase to out-of-phase. Superharmonic resonance of a pendulum with monofrequency excitation had been proposed as a potential model for this phenomenon. In this study, an alternative model of paired pendulums with multiple-frequency excitations is explored. It was predicted that the occurrence of the concurrent transitions was a function of (1) changes in the magnitude ratio of shoulder accelerations at step and stride frequencies that accompany changes in walking speed and (2) proximity of these frequencies to the natural resonance frequencies of the arms modeled as a pair of passive pendulums. Model predictions were compared with data collected from 14 healthy young subjects who were instructed to walk on a treadmill. Walking speeds were manipulated between 0.18 and $1.52 \mathrm{~m} / \mathrm{s}$ in steps of $0.22 \mathrm{~m} / \mathrm{s}$. Kinematic data for the arms and shoulders were collected using a 3D motion analysis system, and simulations were conducted in which the movements of a double-pendulum system excited by the accelerations at the suspension point were analyzed to determine the extent to which the arms acted as passive pendulums. It was confirmed that the acceleration waveforms at the shoulder are composed primarily of stride and step frequency components. Between the shoulders, the stride frequency components were out-of-phase, while the step frequency components were in-phase. The amplitude ratio of the acceleration waveform components at the step and stride frequencies changed as a function of walking speed and were associated with the occurrence of the transitions. Simulation results using these summed components as excitatory inputs to the double-pendulum system were in agreement with actual transitions in $80 \%$ of the cases. The potential role of state-dependent active muscle contraction at shoulder joints on the occurrence of the transitions was discussed. Due to the tendency of arm
\end{abstract}

Correspondence to: M. Kubo

(e-mail: mkubo@alum.bu.edu,

Tel.: +1-313-7645210, Fax: +1-313-7636283) movements to stay in the vicinity of their primary resonance frequency, these active muscle forces were hypothesized to function as escapements that created limit cycle oscillations at the shoulder's resonant frequency.

Keywords: Gait - Biomechanics - Coordination dynamics

\section{Introduction}

Research on transitions between locomotory movement patterns has focused predominantly on rhythmic movements and has been grounded theoretically in the dynamics of coupled nonlinear oscillators (Haken et al. 1985; Diedrich and Warren 1995). The emphasis in the current study is on the role that biomechanics may play in driving transitions that occur from slow to fast walking. At selfselected and fast walking speeds (approximately 1.5 and $2.4 \mathrm{~m} / \mathrm{s}$, respectively), the upper and lower limbs oscillate at the same frequency and each limb oscillates reciprocally with both the contralateral limb and the ipsilateral limb in the sagittal plane (Murray et al. 1967). At slower walking speeds (less than $0.75 \mathrm{~m} / \mathrm{s}$ ), the frequency ratio of arm swing to leg swing changes from 1:1 to $2: 1$ and is accompanied by a phase transition between the arms from a more out-of-phase pattern to a more or less in-phase pattern (Craik et al. 1976; Webb and Tuttle 1989; van Emmerik and Wagenaar 1996; Wagenaar and van Emmerik 2000). Similar phase transitions with walking speed have been observed between the thoracic and pelvic rotations in the transverse plane (Wagenaar and Beek 1992), providing further support for the hypothesis that biped walking has at least two different speed-specific modes of intersegmental coordination (Wagenaar and van Emmerik 2000).

The dynamics of arm movements during walking have been compared to those of a pair of coupled physical pendulums driven by multiple forcing functions (Elftman 1939; Fernandez-Ballesteros et al. 1965; Jackson et al. 1978). Wagenaar and van Emmerik (2000) found that the observed frequency of arm swing at slow walk- 
ing speeds (about $0.5 \mathrm{~m} / \mathrm{s}$ ) coincided with the estimated resonant frequency of the arm, using a pendulum and spring model (see Holt et al. 1990). Using a simple pendulum model, it was found that both frequency ratio and phase transitions occurred when the stride frequency was decreased to slightly lower than the arm's estimated natural pendular frequency (Webb and Tuttle 1989; Webb et al. 1994). However, it has also been reported that the resonant frequency of the arms does not always coincide with the observed frequency of arm swing, and the arm-leg frequency ratio transitions do not always occur simultaneously on both sides of the body (Wagenaar and van Emmerik 2000; Donker et al. 2001).

Thus, the theoretical basis for concurrent frequency and phase transitions in interlimb coordination remains unclear. One hypothesis is that the arm movements are excited by a monofrequency forcing function (i.e., forcing at the stride frequency) and that the 2:1 frequency ratio arises as the result of superharmonic resonance when the stride frequency drops below the natural pendular frequency of arms (Webb et al. 1994). However, the scope of this proposed mechanism is limited to the frequency ratio transition of the arm and leg movements of one body side and may not be sufficient to account for the concurrent interarm phase transition. An alternative hypothesis, developed in the present paper, is that the concurrent frequency ratio and phase transitions are emergent behaviors of a pair of passive pendulums excited by multiple-frequency forcing functions applied at the arm's suspension points (i.e., the shoulders) generated by the upper trunk movement during walking.

However, regardless of which type of hypothesis is entertained, a necessary first step in accounting for both interarm phase transitions (IAPT) and arm-leg frequency ratio transitions (ALFT) is to characterize the walkingspeed-dependent driving forces at the shoulder. Arm motions during walking are driven at the shoulders by active muscle forces and passive resistive (elastic and frictional) forces, by the acceleration of the arm suspension points (i.e., shoulders) produced by motions of the upper trunk, and by reactive coupling forces induced by motion of remote segments. In the present study, we first measured accelerations at the shoulder and then incorporated them into a model that related these accelerations to movements of the upper trunk. Specifically, we modeled arm swing in the sagittal plane as it is influenced by upper trunk movement, which in turn was viewed as a combination of an oscillation in the transverse plane around the trunk's longitudinal axis at stride frequency and an oscillation in the sagittal plane around the transverse axis at step frequency (Thorstensson et al. 1984; Stokes et al. 1989). We hypothesized that horizontal accelerations at the suspension points (i.e., shoulders) are a summation of out-ofphase accelerations at the stride frequency and in-phase accelerations at the step frequency. Vertical acceleration of the shoulder joints was expected to be in-phase at the step frequency. Since at low speeds the oscillation of the upper trunk in the transverse plane at stride frequency is diminished (Wagenaar and Beek 1992), we also hypothesized that an amplitude ratio, defined as the ratio of the in-phase acceleration waveform amplitude at step frequency to the out-of-phase acceleration waveform amplitude at stride frequency in the transverse plane, would increase at low speeds. In turn, the increased amplitude ratio was hypothesized to induce the concurrent IAPT from out-of-phase to in-phase between arm movements and ALFT from 1:1 to 2:1 frequency ratio between arm and leg movements.

In conjunction with our experimental measurements, we also applied the step and stride frequency components of the experimentally observed accelerations to the suspension points of a model of the arms treated as a pair of passive pendulums and tested whether the model captured the experimentally observed transitions.

\section{Methods}

\subsection{Subjects}

Fourteen healthy young subjects with no walking disability (four men and ten women; age range 22-33 years) participated in this study. Prior to participation, subjects gave informed consent in accordance with the policies of the Boston University Institutional Review Board and the United States human subject guidelines.

\subsection{Procedure}

Infrared-emitting diodes (IREDs) were placed bilaterally on the acromion process, lateral epicondyle, radial styloid process, greater trochanter, lateral condyle, and lateral malleolus. Subjects were asked to walk on a treadmill (Accumil-P, Pacer Fitness Systems, Texas, USA). Treadmill speed was adjusted from 0.18 up to $1.52 \mathrm{~m} / \mathrm{s}$ in increments of $0.22 \mathrm{~m} / \mathrm{s}$, first in ascending order then in descending order. Subjects were allowed to practice walking for about $15-30 \mathrm{~s}$ on the treadmill to get used to the assigned walking speeds. After practice, 3D kinematic data were collected using OPTOTRAK (Northern Digital Inc., Canada) at a sampling rate of $100 \mathrm{~Hz}$ for $30 \mathrm{~s}$ in each experimental condition.

\subsection{Data reduction}

2.3.1 Kinematics. Computations were performed using custom programs written in MATLAB (The Mathworks, Inc., Natick, MA, USA). Raw kinematic data were filtered using a fourth-order Butterworth filter (cutoff frequency $5 \mathrm{~Hz}$ ). Time series of arm and leg angles in the sagittal plane were calculated from the filtered data. Arm and leg angles were defined as the angle formed by the line of gravity and the long axis of upper arm and thigh, respectively. Power spectral density (PSD) functions were calculated from the time series of the angles (FFT length $2^{14}$, Hanning window, Welch method). The frequency with the highest PSD peak was identified as the dominant frequency of oscillation for each segment. Evaluation of the frequency ratio between arm and leg swing during walking was based on the dominant frequency of oscillation for 
each segment (Wagenaar and van Emmerik 2000). Considering the natural fluctuations of movement of each body segment during walking, a frequency ratio from arm to leg between 2.1:1 and 1.9:1 was taken as $2: 1$, and a ratio between 1.1:1 and 0.9:1 was taken as 1:1. Changes in the frequency ratio between 1:1 and 2:1 were referred to as arm-leg frequency transitions (ALFT) in this study (Wagenaar and van Emmerik 2000). Changes in relative phase between arms over different walking speeds were based on the two time series of left and right arm angles. Relative phase computation was based on the Hilbert transformation (Cohen 1995), and the mean value of relative phase was calculated using circular statistics (Batschelet 1981). Changes in relative phase between left and right arm between in-phase (close to $0^{\circ}$ ) and out-of-phase (close to $180^{\circ}$ ) were referred to as interarm phase transitions (IAPT) in this study (van Emmerik and Wagenaar 1996).

2.3.2 Model. During walking, upper trunk movements that generate acceleration at the shoulder joints are of two kinds: (1) transverse (horizontal) plane oscillation around the trunk's longitudinal axis at the stride frequency and (2) sagittal plane (anterior-posterior) oscillation around the hip joint at the step frequency (Thorstensson et al. 1984; Stokes et al. 1989). Upper trunk oscillations in the frontal plane were not taken into consideration. Upper trunk oscillations in the transverse plane generate horizontal linear accelerations of the shoulder joints (Fig. 1a), while upper trunk oscillation in the sagittal plane generates both horizontal and vertical linear accelerations of the shoulder joints (Fig. 1b). The PSD of horizontal and vertical accelerations at each shoulder was calculated (FFT length $2^{14}$, Hanning window, Welch method). The concentrations of power of accelerations at distinct frequencies were expressed as percentages of the area under PSD curve with frequency range from 0 to $50 \mathrm{~Hz}$ and referred to as percentage power. The percentage power of peaks at the stride, step, and superharmonic frequencies was calculated to assess the major frequency components of shoulder joint accelerations (Fig. 2).

Linear acceleration at the shoulder joint was obtained as the second derivative of the horizontal and vertical displacement time series of the shoulder marker in space. The FFT was applied to the observed acceleration frequency component time series $\left(A c c_{h}\right.$ and $\left.A c c_{\mathrm{V}}\right)$, and then the outcome of FFT was normalized so that a unit sinusoid in the time domain corresponded to unit amplitude in the frequency domain. ${ }^{1}$ The amplitudes of each acceleration frequency component $\left(A_{h_{\text {STRIDE }}}, A_{h_{\text {STEP }}}\right.$ and $\left.A_{\mathrm{V}_{\text {STEP }}}\right)$ were estimated from the amplitudes of each frequency's peak in the amplitude-normalized FFT plot.

The horizontal acceleration component $\left(A c c_{h}\right)$ and the vertical acceleration component $\left(A c c_{\mathrm{v}}\right)$ generated at a shoulder were approximated to provide driving inputs to

\footnotetext{
$1 P n=a b s(f f t(x)) * 2 /$ length $(x)$, where $f f t(x)$ is an outcome of FFT of signal $x$, length $(x)$ is the number of samples given by time duration* sample frequency, and $P n$ is a normalized outcome.
}

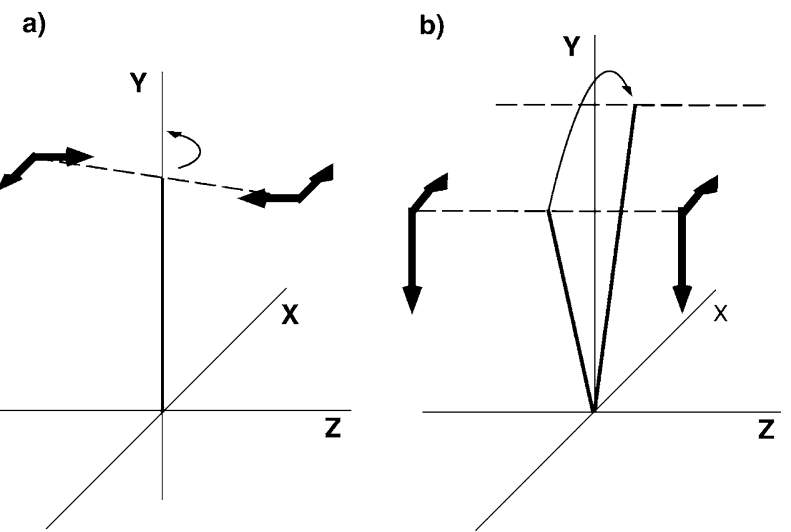

Fig. 1a, b. Models representing the hypothesized acceleration components at the shoulders at stride frequency (a) and step frequency (b). According to the model, horizontal accelerations at the stride frequency are expected to be out-of-phase, while horizontal and vertical accelerations are expected to be in-phase

the model using the following equations:

$A c c_{h} *=A_{h_{\mathrm{STRIDE}}} \cos \left(\varpi t-p_{1}\right)+A_{h_{\mathrm{STEP}}} \cos \left(2 \varpi t-p_{2}\right)$

$A c c_{\mathrm{V}} *=A_{\mathrm{V}_{\mathrm{STEP}}} \cos \left(2 \varpi t-p_{3}\right)$,

where the ${ }^{*}$ superscript denotes the approximating time series, $A_{h_{\text {STRIDE }}}$ and $A_{h_{\text {STEP }}}$ represent the amplitude of the components of the horizontal acceleration time series at the stride frequency $(\varpi)$ and step frequency $(2 \varpi)$, respectively, and $A_{\mathrm{VSTEP}}$ represents the amplitude of the components of the vertical acceleration time series at the step frequency $(2 \varpi)$. Stride frequency $(\omega)$ was defined as the dominant frequency of the leg angle time series. $p_{1}, p_{2}$, and $p_{3}$ represent the initial phases of each acceleration component (i.e., $A_{h_{\text {STRIDE }}}, A_{h_{\text {STEP }}}$, and $A_{\mathrm{V}_{\mathrm{STEP}}}$ ). These phases were estimated by first using zero-phase bandpass digital filtering on the time series of accelerations to extract the acceleration components separately; the initial phases of each component were then determined so that the RMS error between the bandpass-filtered time series and the modeled time series (e.g., $A_{h_{\text {STRIDE }}} \cos \left(\varpi t-p_{1}\right)$ ) became minimum. The passband for stride and step frequency components were $(1 \pm 0.1) \varpi$ and $(1 \pm 0.1) 2 \varpi$, respectively.

Equations (1) and (2) were rewritten using the phase of the fundamental frequency $(\varpi)$ component as a reference phase:

$A c c_{h} *=A_{h_{\mathrm{STRIDE}}} \cos (\varpi t)+A_{h_{\mathrm{STEP}}} \cos \left(2 \varpi t-p_{h}\right)$

$A c c_{\mathrm{V}} *=A_{\mathrm{V}_{\mathrm{STEP}}} \cos \left(2 \varpi t-p_{\mathrm{v}}\right)$,

where $p_{h}=-2 p_{1}+p_{2}$ and $p_{\mathrm{v}}=-2 p_{1}+p_{3}$.

The arms were modeled as two-segment pendulums attached to moving suspension points. The model pendulum consists of two rigid rods with shoulder and elbow joints allowed to oscillate only in the sagittal plane. The physical properties of each segment (length, mass, and moment of inertia) were estimated using the measured anthropometric data of each subject and anthropometric table provided by Dempster et al. (see Winter 1990). Estimation 

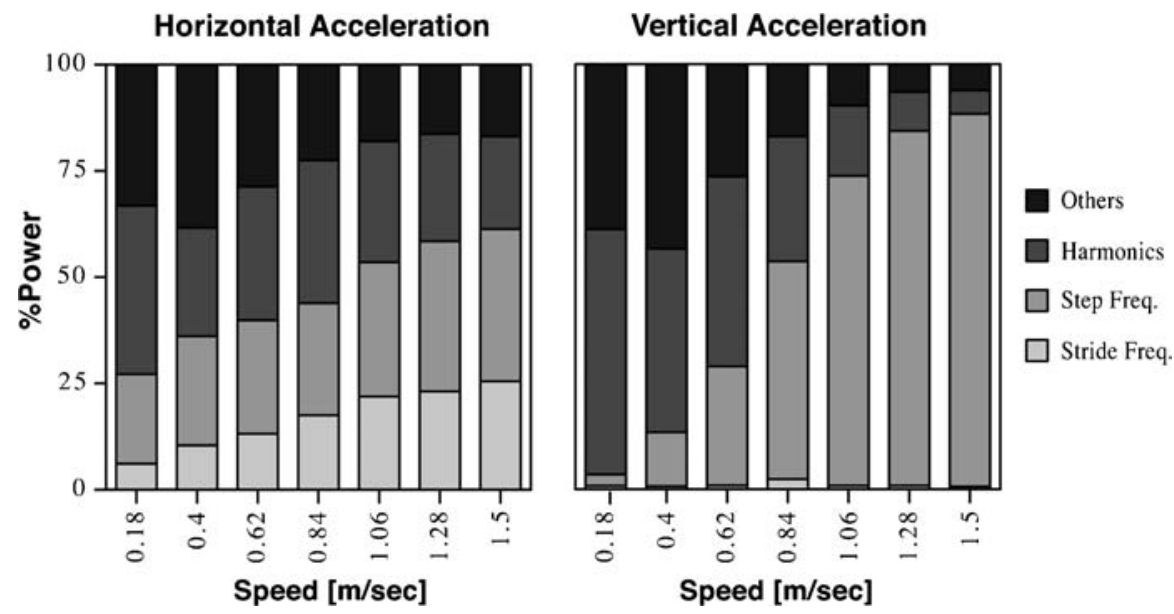

Fig. 2. Changes in mean percentage power of stride frequency, step frequency, superharmonics, and other frequency components in horizontal acceleration (left) and vertical acceleration (right) at shoulder joint over different speed conditions. "Freq" denotes frequency of passive resistive torques due to elasticity and viscosity of soft tissue around shoulder and elbow joints $\left(T_{1 i}\right)$ were adopted from Jackson et al. (1978) and were given by:

$T_{1 i}=\beta\left(-0.9\left(\phi_{i}-\phi_{0 i}\right)-0.1 \dot{\phi}_{i}\right) \quad i=1 \ldots 4$,

where $\phi_{i}$ and $\dot{\phi}_{i}$ represent the angular position and the angular velocity of shoulders $(i=1,2)$ and elbows $(i=$ $3,4)$, respectively, and $\phi_{0 i}$ the equilibrium position of joint $i$ and was set at $0^{\circ}$ for all joints. The value of $\beta$ was set at 1 . The anatomical limit that prevented hyperextension at an elbow joint was expressed as an exponential increase in torque at the elbow joint ( $T_{2 i}$; Jackson et al. 1978) and was given by:

$T_{2 i}=0.1 \mathrm{e}^{\frac{-180}{\pi}\left(\theta_{i}-\theta_{0 i}\right)} \quad i=3,4$,

where $\theta_{i}$ represents the angular position of the elbow joint and $\theta_{0 i}$ is the anatomical limit of range of motion, which was set at 0 in this study. There were no anatomical limits added to the shoulder joints.

2.3.3 Simulation. $\quad A_{c c}{ }^{*}$ and $A c c_{\mathrm{V}}{ }^{*}$ derived from the individual subjects' data were used to specify the acceleration of the suspension points of the subject-specific pendulums in the mathematical model. The equation of motion of the pendulum model for each arm was derived using the Newton-Euler method (Yoshikawa 1990), and the movements of the model pendulum were numerically calculated using the ODE function of MATLAB. The dominant frequency of the "upper arm" oscillation of the model pendulum without forcing was referred to as the natural pendulum frequency. The natural pendulum frequency was determined by the simulation of the model pendulum including passive terms [from (5) and (6)] with initial shoulder angle at $10^{\circ}$ extension and initial elbow angle at $0^{\circ}$ without acceleration at the shoulder suspension point.

Simulation using the forcing functions $A c c_{h}{ }^{*}$ and $A c c_{\mathrm{v}}{ }^{*}$ was started at the resting position of the pendulum, and the measurement of frequency and phase angle was taken for $30 \mathrm{~s}$ following $110 \mathrm{~s}$ of initial simulation.
The dominant frequency of each pendulum and the relative phase between the oscillations of the two pendulums on the right and left sides were evaluated by the same PSD and relative phase analysis used to analyze the observed arm swing during walking.

2.3.4 Statistical analysis. Regression analysis was used to determine how accelerations at the shoulders changed across walking speeds. ANOVA with repeated measures was used to evaluate the effects of walking speed as an intragroup factor (seven levels) on the relative phase of accelerations between shoulder movements. Hysteresis effects in the occurrence of transitions in response to the ascending and descending speed manipulation were evaluated by the Sign Test (Portney and Watkins 1993). Differences in amplitude ratio between the step frequency $\left(A_{h_{\text {STEP }}}\right)$ and stride frequency $\left(A_{h_{\text {STRIDE }}}\right)$ components of the modeled horizontal acceleration time series $\left(A c c_{h}{ }^{*}\right)$ were evaluated before and after ALFTs using the Wilcoxon Signed Rank Test. Differences in the amplitude ratio for asymmetric frequency transitions between arm and leg movements (one body side at 2:1 frequency ratio and the other at 1:1 frequency ratio at the same speed) were also evaluated by the Wilcoxon Signed Rank Test. The agreement between observed and simulated arm swing transitions was evaluated by the Chi-Square Test. Differences in the speed level at which ALFT and IAPT occurred between the observed and simulated data were evaluated by the Wilcoxon Signed Rank Test. All statistical analyses were performed using SAS (SAS Institute, Cary, NC, USA). The level of significance was set at 0.05 .

\section{Results}

\subsection{Observed data}

The summary of physical characteristics of subjects is presented in Table 1. The range of values across speeds of percentage power for stride and step frequency horizontal acceleration components were $6-25 \%$ and $21-35 \%$, respectively. The range of percentage power for the stride frequency vertical acceleration component was $0.6-2.4 \%$, 
Table 1. Physical characteristics of subjects

\begin{tabular}{|c|c|c|c|c|c|c|c|c|}
\hline Subject & Body weight (kg) & Body height (m) & \multicolumn{3}{|l|}{ Upper arm } & \multicolumn{3}{|l|}{ Lower arm ${ }^{\mathrm{a}}$} \\
\hline 1 & 56.7 & 1.58 & 0.20 & 1.59 & $6.62 \mathrm{E}-03$ & 0.24 & 1.25 & $1.57 \mathrm{E}-02$ \\
\hline 2 & 58.5 & 1.7 & 0.21 & 1.64 & $7.34 \mathrm{E}-03$ & 0.26 & 1.29 & $1.86 \mathrm{E}-02$ \\
\hline 3 & 72.6 & 1.71 & 0.23 & 2.03 & $1.07 \mathrm{E}-02$ & 0.26 & 1.60 & $2.28 \mathrm{E}-02$ \\
\hline 4 & 47.6 & 1.64 & 0.22 & 1.33 & $6.48 \mathrm{E}-03$ & 0.26 & 1.05 & $1.49 \mathrm{E}-02$ \\
\hline 7 & 63.5 & 1.62 & 0.20 & 1.78 & 7.53E-03 & 0.26 & 1.40 & $2.05 \mathrm{E}-02$ \\
\hline 8 & 71.2 & 1.71 & 0.22 & 1.99 & $9.70 \mathrm{E}-03$ & 0.27 & 1.57 & $2.47 \mathrm{E}-02$ \\
\hline 9 & 55.8 & 1.63 & 0.18 & 1.56 & $5.23 \mathrm{E}-03$ & 0.23 & 1.23 & $1.41 \mathrm{E}-02$ \\
\hline 10 & 69.6 & 1.73 & 0.23 & 1.95 & $1.04 \mathrm{E}-02$ & 0.26 & 1.53 & 2.29E-02 \\
\hline 11 & 113.4 & 1.78 & 0.28 & 3.18 & $2.64 \mathrm{E}-02$ & 0.33 & 2.49 & $5.95 \mathrm{E}-02$ \\
\hline 12 & 55.5 & 1.65 & 0.20 & 1.55 & 6.61E-03 & 0.24 & 1.22 & $1.59 \mathrm{E}-02$ \\
\hline
\end{tabular}

${ }^{a}$ Lower arm includes forearm and hand

while that for step frequency component was $2.7-87 \%$ (Fig. 2).

The amplitude of horizontal acceleration components at both stride and step frequencies changed as a linear function of walking speed. The amplitude of the vertical acceleration component at step frequency changed as a quadratic function of walking speed, while the vertical stride frequency component remained negligible at all speeds (Fig. 3). Mean intershoulder relative phase of the horizontal acceleration components at stride frequency showed a significant main effect of walking speed $[F(6,77)=22.8, p<0.0001]$. These accelerations were more or less out-of-phase (range: $\left.130-156^{\circ}\right)$ except at the lowest speed $\left(62^{\circ}\right.$ at $0.18 \mathrm{~m} / \mathrm{s}$ ). The mean intershoulder relative phase of the horizontal acceleration components at step frequency was not significantly different across speeds [range: 13 to $\left.30^{\circ} ; F(6,77)=1.1, p=0.37\right]$. The mean intershoulder relative phase of vertical accelerations at step frequency showed a significant main effect of walking speed [range: 3 to $\left.60^{\circ} ; F(6,77)=17.5, p<0.0001\right]$. However, both horizontal and vertical accelerations at step frequency remained close to in-phase across all speeds (Fig. 4).

No significant hysteresis was observed for the speeds across which ALFT occurred during ascending versus descending speed conditions $(n=4, x=1, p=0.31)$. Therefore, subsequent analyses were computed on pooled data in terms of speed conditions. Five subjects out of 14 exhibited ALFT bilaterally at the same speed condition, while others showed either ALFT only at one arm or did not show ALFT at all and maintained a 1:1 frequency ratio (Table 2). The occurrence of these transitions coincided with changes in the amplitude ratio of horizontal acceleration frequency components. In the two consecutive speed conditions across which the ALFT occurred, the transition between 2:1 and 1:1 was accompanied by a significant change $(Z=2.16, p=0.015)$ in the amplitude ratio $(3.18 \pm 2.83$ at the lower speed and $1.94 \pm 1.53$ at the higher speed). In six cases, one arm shows a 2:1 frequency ratio, while the other arm retains a 1:1 ratio at the same speed condition (asymmetric ALFTs). In four out of six asymmetric ALFTs, a significant difference $(Z=2.16, p=0.015)$ in the amplitude ratio was observed for one body side showing a 2:1 ratio and the other showing a $1: 1$ ratio $(1.92 \pm 0.59$ and $0.76 \pm 0.23)$.

\subsection{Simulated data}

In the simulated arm swings, all 14 subjects exhibited ALFT (i.e., ALFT $_{\text {sim }}$ ) at least in one arm (Table 2). The arms' natural pendulum frequency was $0.86 \pm 0.05 \mathrm{~Hz}$

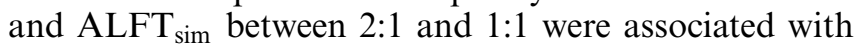
changes in stride frequency between $0.5 \pm 0.1$ and $0.7 \pm$ $0.1 \mathrm{~Hz}$. Fifteen pairs of ALFT sim $_{\text {occurred bilaterally at }}$ the same speeds, accompanied by IAPT from $0.0 \pm 1.4$ to $180.0 \pm 5.4^{\circ}$.

The $\mathrm{ALFT}_{\text {sim }}$ between 2:1 and 1:1 were also accompanied by a significant change $(Z=3.88, p<0.0001)$ in the amplitude ratio of horizontal accelerations $(2.54 \pm 2.0$ and $1.36 \pm 0.78$, respectively). In 11 asymmetric ALFT $_{\text {sim }}$, there was a significant difference $(Z=3.55, p=0.0002)$ in the amplitude ratio between arms ( $2.24 \pm 0.78$ for $2: 1$ and $0.95 \pm 0.44$ for $1: 1)$.

The occurrence or not of ALFTs in subjects was reproduced in simulations for $80 \%$ of the cases. (DF $=1, \chi^{2}=$ 8.9, $p<0.003$; Table 3). Simulated transitions occurred across the same speeds as actual transitions in 21 cases and at one speed increment higher or lower in 18 of the remaining transitions. Overall, however, simulated transitions occurred across significantly higher walking speeds than observed transitions $(Z=-3.4, p<0.001$; Table 4$)$.

\section{Discussion}

The present study has focused on the biomechanical factors that underlie arm-leg frequency ratio transi- 

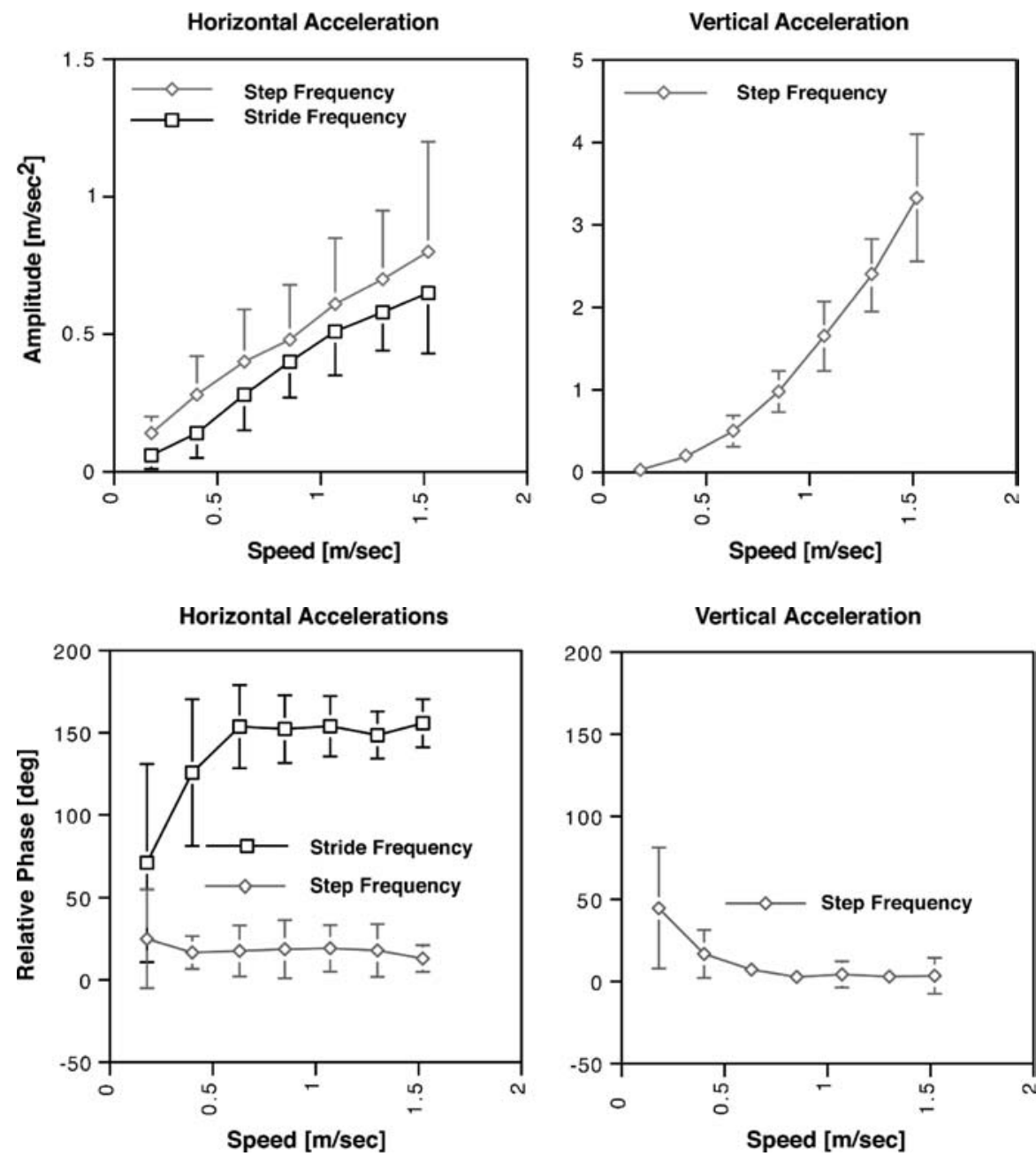

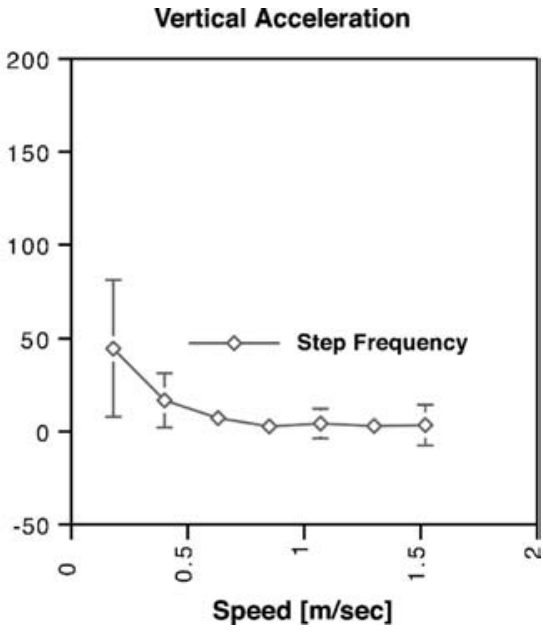

Fig. 3. Left panel: Changes in the amplitudes of horizontal shoulder acceleration with (a) stride frequency and (b) step frequency. Both accelerations change as a linear function of walking speeds $\left(y=0.46 x-0.017, r^{2}=0.99\right.$, $p<0.0001$ and $y=0.484 x+0.076$, $r^{2}=0.99, p<0.0001$, respectively). Right panel: The amplitude of vertical shoulder acceleration at step frequency changes as a quadratic function of walking speeds $\left(y=1.531 x^{2}-0.135 x-0.0002, r^{2}>0.99\right.$, $p<0.0001$ ), whereas the stride frequency component remains negligible across all walking speeds
Fig. 4. Left panel: Changes in the relative phase between accelerations between left and right shoulder movements. Right panel: Horizontal accelerations at stride frequency remain close to out-of-phase at all but the lowest walking speeds. Both horizontal and vertical accelerations at step frequency remain close to in-phase for all but the lowest walking speeds
Table 2. Number of ALFTs for a subject

\begin{tabular}{lllllll}
\hline & No. of ALFTs & 4 & 3 & 2 & 1 & 0 \\
\hline \multirow{2}{*}{ No. of subjects } & Actual & 7 & 4 & 1 & 0 & 2 \\
& Simulated & 11 & 1 & 2 & 0 & 0 \\
\hline
\end{tabular}

Because of the experimental design used in the present study, each subject could have shown four ALFTs (ascending-descending speeds, left-right sides)

Table 3. Occurrence of ALFT between 1:1 and 2:1 in actual and simulated arm swing

\begin{tabular}{lll}
\hline & \multicolumn{2}{l}{ Actual } \\
\cline { 2 - 3 } & Transition & No transition \\
\hline Simulated & & \\
Transition & 41 & 10 \\
No transition & 1 & 4 \\
\hline
\end{tabular}

In the cases of "No Transition", ALFT remains 1:1

tions (ALFT) and interarm phase transitions (IAPT) in response to changes in walking speed. Accelerations acting at the shoulders were modeled as those due to transverse and sagittal oscillations of the upper trunk.
Table 4. Comparison of speed level at which the ALFT occurred between observed and simulated arm swing

\begin{tabular}{llllllll}
\hline Difference in speed level & -2 & -1 & 0 & +1 & +2 & +3
\end{tabular}

$\begin{array}{lllllll}\text { Number of observations } & 0 & 1 & 21 & 17 & 2 & 0\end{array}$

Unit of speed level is $0.22 \mathrm{~m} / \mathrm{s}$

When the simulated ALFT occurred at a higher speed range by one level (i.e., $0.22 \mathrm{~m} / \mathrm{s}$ ) than corresponding observed ALFT, it was shown as +1 in the table

Transverse accelerations at the shoulder were found to be comprised of both step and stride acceleration components, step components being more in-phase and stride components being more out-of-phase. Both horizontal and vertical accelerations of the shoulder joints exhibited frequency and phase characteristics predicted by this model of upper trunk movement during walking. The linear increase of both frequency components of the horizontal accelerations $\left(A_{h_{\text {STRIDE }}}\right.$ and $\left.A_{h_{\text {STEP }}}\right)$ and the quadratic increase of the step frequency component of the vertical acceleration $\left(A_{\mathrm{V}_{\mathrm{STEP}}}\right)$ also support the simple model of upper trunk movement used in this study.

Occurrences of concurrent arm-leg frequency ratio transitions (ALFT) and interarm phase transitions (IAPT) were associated with significant changes in the 
amplitude ratio between $A_{h_{\text {STEP }}}$ and $A_{h_{\text {STRIDE }}}$ that accompanied changes in walking speed. In both real and simulated arm swings, symmetric concurrent transitions with decreasing walking speed (i.e., ALFT from 1:1 to 2:1 and IAPT from out-of-phase to in-phase) were associated with significant increases in the amplitude ratios (i.e., $A_{h_{\text {STEP }}} / A_{h_{\text {STRIDE }}}$ ). Occurrences of asymmetric ALFTs reported by Wagenaar and van Emmerik (2000) and Donker et al. (2001) may be accounted for by the significant difference of the amplitude ratios between shoulders. The increase in the amplitude ratio at lower walking speeds may be due primarily to a decrease in $A_{h_{\text {STRIDE }}}$, a finding consistent with the observation that the upper and lower trunk demonstrate minimal counterrotation at slow walking speeds (Wagenaar and Beek 1992; van Emmerik and Wagenaar 1996).

In addition to the amplitude ratios of accelerations, the relative proximity of step or stride frequency to the natural pendulum frequency of the arm may be a second determinant of the occurrence of the concurrent transitions. Results of the simulated arm swing are in agreement with the observation that ALFTs occur when the stride frequency becomes lower than the natural pendulum frequency of the arm (Webb and Tuttle 1989; Webb et al. 1994). One interpretation is that ALFT and IAPT are related to the competition between the magnet effect of the driving excitation frequency and maintenance tendency of a pendulum to stay at its primary resonance (von Holst 1973). The lower stride frequency that accompanies lower walking speeds results in acceleration frequencies at the shoulder that are further from the natural frequency of the arms than are the frequency of accelerations at the two shoulders produced at the step frequency. The capability of the arms to be entrained to the stride frequency is lost and is replaced by the capability to entrain to step frequency. Since the accelerations due to step frequency are caused by anterior-posterior oscillations of the trunk in the sagittal plane, they are in-phase. Therefore, switching from one frequency component of excitations to the other causes transitions in both the frequency ratio between arm and leg movements and the phase relation between left and right arm movements.

In about half the simulations, transitions occurred at one speed higher than those in observed arm swing. This may be due to an error in estimating the resistive torque resulting from elasticity and friction of soft tissues around joints. However, Jackson et al. (1978) manipulated the value of $\beta$ [see (5)] in his study and reported that the simulated arm movement was not significantly different from the observed arm movement when the $\beta$ value was within a range between 0.1 and 10 . Therefore, any error in this parameter may have had only minor impact.

More likely is the possibility that the discrepancy may be accounted for by the contribution of active muscle contraction, which was not included in the model in this study. Increased muscle activity around the shoulder joints occurs in response to transverse rotation of the upper trunk even when the arms are bound to the trunk (Fernandez-Ballesteros et al. 1965). This trunk-rotationinduced muscle activity may provide an additional state- dependent forcing function at the stride frequency that is out-of-phase between shoulders, resisting the ALFT from 1:1 to 2:1 and IAPT from out-of-phase to in-phase with decreasing walking speed. It may be that failures and delays in transitions in observed arm swings as speed decreases are due to a strong muscular input in some subjects. Models that include this hypothesized input may result in better simulations. Experimentally it might be useful to assess the active contribution of muscle in some subjects using EMG. Nevertheless, the finding that the model successfully simulates transitions in 39 of 46 transitions within one walking speed increment (Table 4) provides encouraging support for the viability of our approach.

In summary, the basic mechanism of the frequency ratio and the concurrent phase transition between arms and legs observed at slow walking speeds can, to a great extent, be accounted for as the behavior of a pair of pendulums driven by multiple-frequency excitation resulting from upper trunk movement. Although superharmonic resonance may account for frequency ratio transitions between $2: 1$ and $1: 1$, this approach does not eliminate the possibility of a combination of a 2:1 frequency ratio and an out-ofphase interarm coordination. This combination was never observed in the data or simulations. The current model has the advantage of accounting for both ALFT and IAPT. Out-of-phase active muscle contractions between shoulders, not explicitly included in the model, may have contributed to the shift of transition point to in-phase arm swing in simulation as walking speed decreased.

This study used a new approach to understanding phase transitions in walking in which we combined multiple forcing functions applied to the shoulders and the pendular dynamics of the arms. Previous research has attempted to understand phase transitions within the context of pendular dynamics, entrainment, or monofrequency forcing functions and superharmonic resonance. Our approach was successful in predicting both phase and frequency transitions in the majority of cases and for understanding asymmetric transitions. None of the previous models has been able to succeed on all three criteria. Further research is needed to gain a better understanding of the cases where the model fails. In particular, we believe that the basic model will need to be refined to account for the speed differences in transitions between observed and predicted data. Overall, we suggest that the results provide evidence of the manner in which interlimb coordination patterns are related to the pendular characteristics of arm movements and the forces acting on the arms produced by motions of the upper trunk.

\section{References}

Batschelet E (1981) Circular statistics in biology. Academic, New York

Cohen L (1995) Time-frequency analysis. Prentice-Hall, PTR, Upper Saddle River, NJ

Craik R, Herman RM, Finlay FR (1976) Human solutions for locomotion: inter limb coordination. In: Herman RM, 
Grillner S, Stein PSG, Stuart DG (eds) Neural control of locomotion. Plenum, New York, pp 51-63

Diedrich FJ, Warren WH (1995) Why change gaits? Dynamics of the walk-run transition. J Exp Psychol Hum 21: 183-202

Donker SF, Beek PJ, Wagenaar RC, Mulder T (2001) Coordination between arm and leg movements during locomotion. J Mot Behav 33:86-102

Elftman H (1939) The function of the arms in walking. Hum Biol 11:529-535

Fernandez-Ballesteros ML, Buchthal F, Rosenfalck P (1965) The pattern of muscular activity during the arm swing of natural walking. Acta Physiol Scand 63:296-310

Haken H, Kelso JAS, Bunz H (1985) A theoretical model of phase transitions in human hand movements. Cybernetics 51:347-356

Holt KG, Hamill J, Andres RO (1990) The force driven harmonic oscillator as a model for human locomotion. Hum Mov Sci 9:55-68

Jackson KM, Joseph J, Wyard SJ (1978) A mathematical model of arm swing during human locomotion. J Biomech 11: 277-289

Murray MP, Sepic SB, Barnard EJ (1967) Patterns of sagittal rotation of the upper limbs in Walking. Phys Ther 47: 272-284

Portney LG, Watkins MP (1993) Foundations of clinical research. Appleton \& Lange, Norwalk, CT
Stokes VP, Andersson C, Forssberg H (1989) Rotational and translational movement features of the pelvis and thorax during adult human locomotion. J Biomech 22:43-50

Thorstensson A, Nilsson J, Carlson H, Zomlefer R (1984) Trunk movements in human locomotion. Acta Physiol Scand 121:9-22

van Emmerik RE, Wagenaar RC (1996) Effects of walking on relative phase dynamics in the trunk in human walking. J Biomech 29:1175-1184

von Holst E (1973) The behavioral physiology of animal and man. University of Miami Press, Coral Gables, FL

Wagenaar RC, Beek WJ (1992) Hemiplegic gait: a kinematic snalysis using walking speed as a basis. J Biomech 25: $1007-1015$

Wagenaar RC, van Emmerik REA (2000) Resonant frequency of arms and legs identify different walking patterns. J Biomech 33:853-861

Webb D, Tuttle R (1989) The effects of stride frequency on the motion of the upper limbs in human walking. Am J Phys Anthropol 78:321-322

Webb D, Tuttle R, Baksh M (1994) Pendular activity of human upper limbs during slow and normal walking. Am J Phys Anthropol 93:477-489

Winter DA (1990) Biomechanics and motor control of human movement. Wiley, New York

Yoshikawa T (1990) Foundation of robotics. MIT Press, Cambridge, MA 